\title{
Gastrointestinal involvement in patients with vasculitis: IgA vasculitis and eosinophilic granulomatosis with polyangiitis
}

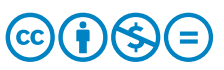

\section{Authors}

Keisuke Kawasaki ${ }^{1}$, Shotaro Nakamura1 ${ }^{1}$ Motohiro Esaki²,3, Koichi Kurahara ${ }^{4}$, Makoto Eizuka ${ }^{5}$, Yasuharu Okamoto ${ }^{3}$, Takashi Hirata ${ }^{4}$, Minako Hirahashi ${ }^{6}$, Yumi Oshiro7, Shunichi Yanai ${ }^{1}$, Kunihiko Sato ${ }^{1}$, Yosuke Toya ${ }^{1}$, Makoto Maemondo $^{8}$, Yasuo Terayama9 ${ }^{9}$, Tamotsu Sugai ${ }^{5}$, Takayuki Matsumoto ${ }^{1}$

Institutions

1 Division of Gastroenterology, Iwate Medical University, Morioka, Japan

2 Department of Endoscopic Diagnostics and Therapeutics, Saga University Hospital, Saga, Japan

3 Department of Medicine and Clinical Science, Graduate School of Medical Sciences, Kyushu University, Fukuoka, Japan

4 Division of Gastroenterology, Matsuyama Red Cross Hospital, Matsuyama, Japan

5 Department of Diagnostic Pathology, Iwate Medical University, Morioka, Japan

6 Department of Anatomic Pathology, Graduate School of Medical Sciences, Kyushu University, Fukuoka, Japan

7 Department of Pathology, Matsuyama Red Cross Hospital, Matsuyama, Japan

8 Division of Pulmonary Medicine, Allergy, and Rheumatology, Iwate Medical University, Morioka, Japan

9 Division of Neurology and Gerontology, Department of Internal Medicine, Iwate Medical University, Morioka, Japan

submitted 26.2.2019

accepted after revision 29.5.2019

Bibliography

DOI https://doi.org/10.1055/a-0977-2777 |

Endoscopy International Open 2019; 07: E1333-E1343

(c) Georg Thieme Verlag KG Stuttgart · New York

eISSN 2196-9736

Corresponding author

Keisuke Kawasaki, MD, Division of Gastroenterology,

Department of Internal Medicine, Iwate Medical University, 19-1, Uchimaru, Morioka, 020-8505, Japan

Fax: +81-19-652-6664

kkeisuke@iwate-med.ac.jp

\section{ABSTRACT}

Background and study aims Among vasculitides, IgA vasculitis (IgAV) and eosinophilic granulomatosis with polyangiitis (EGPA) frequently damage the gastrointestinal tract. However, only a few studies have investigated the entire gastrointestinal tract in patients with IgAV or EGPA by endoscopy. The aim of this study was to clarify endoscopic characteristics of patients with IgAV and those with EGPA. Patients and methods Clinicopathological and endoscopic findings were retrospectively compared between 33 patients with IgAV and 19 patients with EGPA.

Results Gastrointestinal involvement was observed in 33 patients with IgAV (100\%) and in 8 patients with EPGA (42\%; $P=0.0001)$. Duodenal involvement was more frequent in patients with IgAV $(75.8 \%)$ than in those with EGPA (21.1\%, $P=0.0002)$. Jejunoileal involvement was frequent in both groups (IgAV 94.4\%; EGPA 77.8\%). Gastric mucosal erythema was more frequent in patients with IgAV $(18.2 \%)$ than in those with EGPA ( $0 \%, P=0.0481)$. Duodenal mucosal erythema (IgAV 54.6\%; EGPA $21.1 \%, P=0.0227$ ), ulcer (IgAV $33.3 \%$; EGPA $0 \%, P=0.0041$ ), and hematomalike protrusion (IgAV $21.1 \%$; EGPA $0 \%, P=0.039$ ) were more frequently observed in patients with IgAV than in those with EGPA.

Conclusions Frequent duodenal involvement, gastric mucosal erythema, and duodenal lesions including erythema, ulcer, and hematoma-like protrusion are characteristic of patients with IgAV. Because jejunoileal involvement was frequent in both groups of patients, small-bowel endoscopies should be performed for diagnosis of small-bowel lesions in patients with IgAV and EGPA. 


\section{Introduction}

Vasculitis is inflammation of blood vessel walls and its extent and clinical course depend on the size and location of the affected vessels [1]. Vasculitides can be classified into large-, medium- and small-sized vessel vasculitis according to the Chapel Hill Consensus Conference on the Nomenclature of Vasculitidies [2]. Vasculitis affects various organs including kidneys, skin, joints, lungs, and the gastrointestinal tract. Among these organs injury, gastrointestinal involvement can manifest as severe problems such as inflammation, ischemia, hemorrhage, obstruction, or perforation [3].

Among vasculitides, IgA vasculitis (IgAV) and eosinophilic granulomatosis with polyangiitis (EGPA) frequently damage the gastrointestinal tract [4-6]. Endoscopic characteristics of IgAV or EGPA have been described in the literature [7-10]. To date, however, only a few studies have investigated the entire gastrointestinal tract in patients with IgAV or EGPA by endoscopy. Furthermore, no previous studies have compared endoscopic findings between patients with IgAV and EGPA.

We conducted a retrospective analysis to compare clinical, endoscopic and pathological characteristics between patients with IgAV and those with EGPA whose entire gastrointestinal tract was examined endoscopically.

\section{Patients and methods}

\section{Study population}

The current investigation was based on retrospective data collection. We reviewed medical records at Iwate Medical University, Kyushu University, and Matsuyama Red Cross Hospital from 2008 to 2017 and identified all patients with a diagnosis of

IgAV or EGPA. Among those patients, we excluded patients in whom gastrointestinal endoscopy was not performed. The study protocol was approved by the Institutional Review Board at Iwate Medical University, Kyushu University, and Matsuyama Red Cross Hospital. The study was conducted in accordance with the Helsinki Declaration (6th revision, 2008).

\section{Data collection}

Demographics of study subjects were extracted via chart review. Evaluated characteristics included age, sex, allergic diseases (bronchial asthma or allergic rhinitis), laboratory data, organopathy, endoscopic findings, histologic findings, and treatment. Laboratory assessments included white blood cell (WBC), eosinophil, hemoglobin, platelet, serum creatinine and C-reactive protein (CRP), and urinalysis to screen for hematuria and proteinuria before initial treatment. Organopathy included skin involvement (purpura or urticaria), lung involvement (lung infiltrate, nodules or pleural effusion on chest radiograph or computed tomography), neurologic involvement, muscle involvement, and gastrointestinal involvement (abdominal pain, hematemesis, melena, hematochezia, nausea/vomiting, diarrhea and constipation).

The upper gastrointestinal tract was examined by esophagogastroduodenoscopy (EGD). The jejunum and/or ileum was examined by capsule endoscopy (CE) or balloon-assisted endos- copy (BAE). The colorectum was examined by colonoscopy. Endoscopic examination was performed when patients manifested clinical symptoms suggestive of gastrointestinal involvement, such as abdominal pain, hematemesis, melena, hematochezia, nausea, vomiting, diarrhea and constipation, or when they manifested signs of gastrointestinal bleeding including hematemesis, melena, hematochezia, and anemia (a hemoglobin level $<10 \mathrm{~g} / \mathrm{dL}$ ). We also performed endoscopy for asymptomatic patients in consideration of evaluation of subclinical gastrointestinal involvement or when they had an indication for use of steroids. Endoscopic examinations were performed within 14 days after onset of IgAV or EGPA. Endoscopic findings included mucosal erythema, erosion, ulcer, and/or hematoma-like protrusion.

Characteristic histological findings of IgAV were defined as small vessel vasculitis with polymorphonuclear leucocyte infiltration (leukocytoclastic vasculitis), while those of EGPA were defined as small and medium-sized vessel vasculitis, eosinophilic infiltrates, and/or extravascular granulomas [4,11]. As for treatment, steroid, cyclophosphamide, and/or intravenous immunoglobulin (IVIG) were used. We treated patients with intravenous or oral proton pump inhibitor (PPI) when they had upper gastrointestinal lesions or they were treated with a steroid.

\section{IgAV and EGPA diagnosis}

Diagnosis of IgAV and EGPA was based on American College of Rheumatology 1990 criteria $[4,12,13]$. We defined IgAV if patients had two or more of the following four criteria: age 20 or less at disease onset; palpable purpura; acute abdominal pain; and biopsy showing granulocytes in the walls of small arterioles or venules. Also, we defined EGPA if patients have four or more of the following six criteria: asthma; eosinophilia greater than $10 \%$ on differential WBC count; mononeuropathy (including multiplex) or polyneuropathy; non-fixed pulmonary infiltrates on roentgenography; paranasal sinus abnormality; and biopsy containing a blood vessel with extravascular eosinophils.

\section{Statistical analysis}

Parametric data are expressed as mean \pm SD. Nonparametric data are expressed as numbers and percentages. Comparisons between any two groups were performed by the Mann-Whitney test or chi-squared test where appropriate. $P<0.05$ was considered to be significant. All statistical computations were performed with JMP version 13 (Statistical Discovery Program, Cary, North Carolina, United States).

\section{Results}

\section{Clinical features and laboratory data on IgAV and EGPA}

Fifty-two patients who had a diagnosis of IgAV or EGPA (33 IgAV; 19 EGPA). - Table 1 compares clinical and laboratory characteristics between the two groups. There was no significant difference between them groups in terms of age, gender, hemoglobin level, platelet count, creatinine level, CRP, or treatment by steroid. 
- Table 1 Clinical and laboratory characteristics between patients with IgAV and those with EGPA (\%).

\begin{tabular}{|c|c|c|c|}
\hline & IgAV patients $(n=33)$ & EGPA patients $(n=19)$ & $P$ value \\
\hline Age & $46.4 \pm 24.7$ & $56.4 \pm 15.3$ & 0.2061 \\
\hline \multicolumn{3}{|l|}{ Gender } & \multirow[t]{3}{*}{0.5737} \\
\hline - Male & $16(48.5)$ & $11(57.9)$ & \\
\hline - Female & $17(51.5)$ & $8(42.1)$ & \\
\hline \multicolumn{4}{|l|}{ Allergic diseases } \\
\hline - Bronchial asthma & $2(6.1)$ & $19(100)$ & 0.0001 \\
\hline - Allergic rhinitis & $0(0)$ & $4(21.1)$ & 0.0143 \\
\hline \multicolumn{4}{|l|}{ Laboratory data } \\
\hline - WBC count, $/ \mu \mathrm{L}$ & $11505 \pm 5295$ & $24076 \pm 5904$ & 0.0001 \\
\hline - Eosinophil count, $/ \mu \mathrm{L}$ & $178 \pm 165$ & $13824 \pm 6792$ & 0.0001 \\
\hline - Hemoglobin, g/dL & $13.5 \pm 2.0$ & $13.1 \pm 1.7$ & 0.3467 \\
\hline - Platelet count, / $/ \mathrm{L}$ & $268212 \pm 95444$ & $297000 \pm 93761$ & 0.3184 \\
\hline - Serum creatinine, mg/dL & $1.0 \pm 0.9$ & $1.0 \pm 1.1$ & 0.7466 \\
\hline . CRP, mg/dL & $4.5 \pm 6.8$ & $5.6 \pm 5.4$ & 0.1898 \\
\hline - Positive urine occult blood & $28(84.8)$ & $3(15.8)$ & 0.0001 \\
\hline - Positive urine protein & $27(81.8)$ & $7(36.8)$ & 0.0042 \\
\hline \multicolumn{4}{|l|}{ Site of involvement } \\
\hline - Skin & $33(100)$ & $10(52.6)$ & 0.0001 \\
\hline - Lung & $0(0)$ & $13(68.4)$ & 0.0001 \\
\hline - Nerve & $0(0)$ & $16(84.2)$ & 0.0001 \\
\hline - Muscle & $0(0)$ & $6(31.6)$ & 0.0013 \\
\hline - Gastrointestinal tract & $33(100)$ & $8(42.1)$ & 0.0001 \\
\hline \multicolumn{4}{|l|}{ Treatment } \\
\hline " Steroid & $32(97)$ & $19(100)$ & 1 \\
\hline - Cyclophosphamide & $0(0)$ & $3(15.8)$ & 0.0438 \\
\hline . IVIG & $0(0)$ & $11(57.9)$ & 0.0001 \\
\hline - PPI & $32(97)$ & $19(100)$ & 1 \\
\hline
\end{tabular}

Patients with EGPA more frequently had bronchial asthma (100\%) and allergic rhinitis $(21.1 \%)$ than patients with IgAV (6.1\% and $0 \%$, respectively). WBC and eosinophil counts in the peripheral blood at disease onset were significantly higher in patients with EGPA $(24,076 \pm 5,904 / \mu \mathrm{L}$ and $13,824 \pm 6,792 / \mu \mathrm{L})$ than in those with $\operatorname{IgAV}(11,505 \pm 5295 / \mu \mathrm{L}$ and $178 \pm 165 / \mu \mathrm{L}$, respectively). Conversely, rates of microhematuria and proteinuria were higher in patients with IgAV (84.8\% and $81.8 \%$ ) than in those with EGPA ( $15.8 \%$ and $36.8 \%$, respectively).

Regarding symptoms of organopathy, the rate of skin involvement and gastrointestinal involvement were higher in patients with IgAV (100\% and $100 \%$, respectively) than in those with EGPA (52.6\% and $42.1 \%$, respectively), whereas rates of involvement of lung, nerve, and muscle were higher in patients with EGPA (68.4\%, $84.2 \%$ and $31.6 \%$, respectively) than in those with IgAV (all none).

As for treatments, 51 of 52 patients (98\%) were treated with steroids and PPI ( $\triangleright$ Table 1 ). Cyclophosphamide and IVIG were administered to several patients with EGPA and none of those with IgAV. 


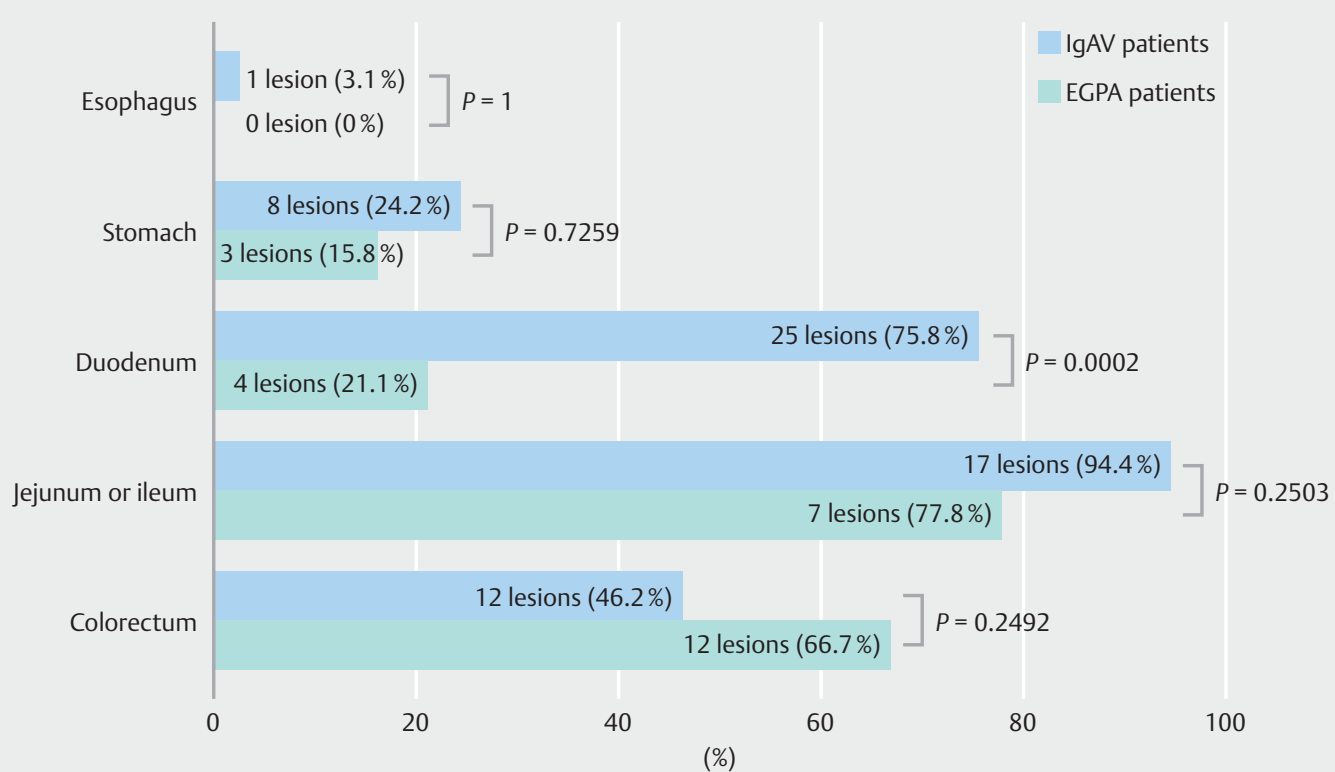

IgAV: IgA vasculitis, EGPA: eosinophilic granulomatosis with poliangiitis

Fig. 1 Frequency of lesions see on endoscopy. Esophagus, stomach, and duodenum were observed in 33 IgAV patients and 19 EGPA patients, the jejunum or ileum was observed in $18 \mathrm{IgAV}$ patients and 9 EGPA patients, and the colorectum was observed for $26 \mathrm{IgAV}$ patients and 18 EGPA patients. IgAV patients had more lesions in the duodenum than did EGPA patients (75.8\% of IgAV; $21.1 \%$ of EGPA; $P=0.0002)$.

\section{Frequency of endoscopic abnormalities}

EGD was performed in all 52 patients. CE or BAE was performed in 27 patients (IgAV 18; EGPA 9). Colonoscopy was performed in 44 patients (IgAV 26; EGPA 18). - Fig. 1 shows frequency of lesions detected by endoscopy. Patients with IgAV had duodenal lesions more frequently than those with EGPA (75.8\% vs. $21.1 \%$; $P=0.0002)$. No significant difference was observed between patients with IgAV and those with EGPA regarding frequency of lesions in the esophagus, stomach, jejunum/ileum or colorectum.

\section{EGD findings}

EGD findings in patients with IgAV and EGPA are shown in $>\mathbf{T a -}$ ble 2 and $>$ Fig. 2. In the esophagus, ulcer was observed only in one patient with IgAV, and other findings were not seen in both groups. In the stomach, mucosal erythema was seen in six of 33 patients (18.2\%) with IgAV and none of 19 patients with EGPA $(P=0.0481)$. Incidence of other gastric lesions and location of each lesion in the stomach did not differ between the two groups (upper/middle/lower, 7/4/2 in IgAV and 3/0/0 in EGPA).

In the duodenum, mucosal erythema (IgAV 54.6\%; EGPA $21.1 \%, P=0.0227$ ), ulcer (IgAV 33.3\%; EGPA 0\%, $P=0.0041)$, and hematoma-like protrusion (IgAV $21.1 \%$; EGPA $0 \%, P=$ 0.039 ) were observed more frequently in patients with IgAV than in those with EGPA. Incidence of erosion was no different between the two groups. Lesions were more frequently located on the second portion of the duodenum in patients with IgAV (bulb/2nd portion, 12/22) than in those with EGPA (bulb/2nd portion, 3/4; $P=0.0034)$. All patients with IgAV or EGPA who had EGD findings had multiple lesions.

\section{CE or BAE findings}

CE and/or BAE findings in patients with IgAV and EGPA are shown in > Table 2 and $>$ Fig. 3 . Incidence of mucosal erythema, erosion, ulcer or hematoma-like protrusion did not differ between two groups. All patients with IgAV or EGPA who had CE or BAE findings had multiple lesions. Lesion location was no different between the two groups (jejunum/ileum, 17/15 in IgAV; $7 / 7$ in EGPA).

\section{Colonoscopic findings}

Colonoscopic findings in patients with IgAV and EGPA are shown in > Table 2 and $>$ Fig. 4. Incidence of mucosal erythema, erosion, ulcer or hematoma-like protrusion did not differ between the two groups. Lesion location was no different between the two groups (cecum to transverse colon/descending colon to rectum, 10/10 in IgAV; 4/12 in EGPA).

\section{Endoscopic findings in patients with gastrointestinal bleeding}

Symptoms and endoscopic findings in patients with gastrointestinal bleeding are shown in $>$ Table 3 . There were nine patients with IgAV and two patients with EGPA who manifested gastrointestinal bleeding. Seven patients had hematochezia, two patients had melena and anemia, and one patient had hematemesis. Under EGD, one patient had esophageal lesions, three patients had gastric lesions, and six patients had duode- 
- Table 2 Endoscopic findings in patients with IgAV and EGPA (\%).

\begin{tabular}{|c|c|c|c|}
\hline & IgAV patients & EGPA patients & $P$ value \\
\hline EGD findings & $n=33$ & $n=19$ & \\
\hline \multicolumn{4}{|l|}{ Esophagus } \\
\hline - Mucosal erythema & $0(0)$ & $0(0)$ & 0 \\
\hline - Erosion & $0(0)$ & $0(0)$ & 0 \\
\hline - Ulcer & $1(3.0)$ & $0(0)$ & 1 \\
\hline - Hematoma-like protrusion & $0(0)$ & $0(0)$ & 0 \\
\hline \multicolumn{4}{|l|}{ Stomach } \\
\hline - Mucosal erythema & $6(18.2)$ & $0(0)$ & 0.0481 \\
\hline - Erosion & $5(15.2)$ & $2(10.5)$ & 1 \\
\hline - Ulcer & $2(6.1)$ & $1(5.3)$ & 1 \\
\hline - Hematoma-like protrusion & $1(3.0)$ & $0(0)$ & 1 \\
\hline \multicolumn{4}{|l|}{ Duodenum } \\
\hline " Mucosal erythema & $18(54.6)$ & $4(21.1)$ & 0.0227 \\
\hline - Erosion & $2(6.1)$ & $4(21.1)$ & 0.1751 \\
\hline - Ulcer & $11(33.3)$ & $0(0)$ & 0.0041 \\
\hline - Hematoma-like protrusion & $7(21.1)$ & $0(0)$ & 0.039 \\
\hline CE or BAE findings & $\mathrm{n}=18$ & $n=9$ & \\
\hline - Mucosal erythema & $13(72.2)$ & $3(33.3)$ & 0.0969 \\
\hline - Erosion & $10(55.6)$ & $4(44.4)$ & 0.6946 \\
\hline - Ulcer & $10(55.6)$ & $5(55.6)$ & 1 \\
\hline - Hematoma-like protrusion & $3(16.7)$ & $0(0)$ & 0.5292 \\
\hline Colonoscopic findings & $n=26$ & $n=18$ & \\
\hline - Mucosal erythema & $10(38.5)$ & $9(50)$ & 0.5419 \\
\hline - Erosion & $8(30.8)$ & $9(50)$ & 0.225 \\
\hline - Ulcer & $6(23.1)$ & $3(16.7)$ & 0.7161 \\
\hline - Hematoma-like protrusion & $4(15.4)$ & $0(0)$ & 0.1327 \\
\hline
\end{tabular}

nal lesions. CE or BAE was performed in seven patients, all of whom had small bowel lesions. Of nine patients examined by colonoscopy, four patients had colorectal lesions. However, none of those 11 patients with gastrointestinal bleeding were treated with endoscopic hemostasis.

\section{Response to therapy in gastrointestinal involvement of IgAV and EGPA}

Follow-up endoscopy was performed in a certain proportion of patients with IgAV or EGPA after treatment. Follow-up endoscopy included EGD in 17 patients (15 patients with IgAV and two patients with EGPA), CE or BAE in seven patients (four patients with IgAV and three patients with EGPA), colonoscopy in 12 patients (seven patients with IgAV and five patients with
EGPA). Follow-up examinations revealed that the preceding lesions had healed or completely disappeared. All patients were treated with a steroid and PPI. Two patients with EGPA and one patient with EGPA were treated with IVIG and cyclophosphamide, respectively.

\section{Characteristic histologic findings in IgAV and EGPA}

Characteristic histologic findings - leukocytoclastic vasculitis, eosinophilic infiltration, small-seized vessel vasculitis, and extravascular granuloma - in IgAV and EGPA are shown in

- Fig. 5. Biopsy specimens were taken from the skin, esophagus, stomach, duodenum, jejunum/ileum and colorectum in 41 (IgAV 31; EGPA 10), 11 (IgAV 3; EGPA 8), 36 (IgAV 23; EGPA 13), 50 (IgAV 35; EGPA 15), 31 (IgAV 21; EGPA 10), and 35 (IgAV 

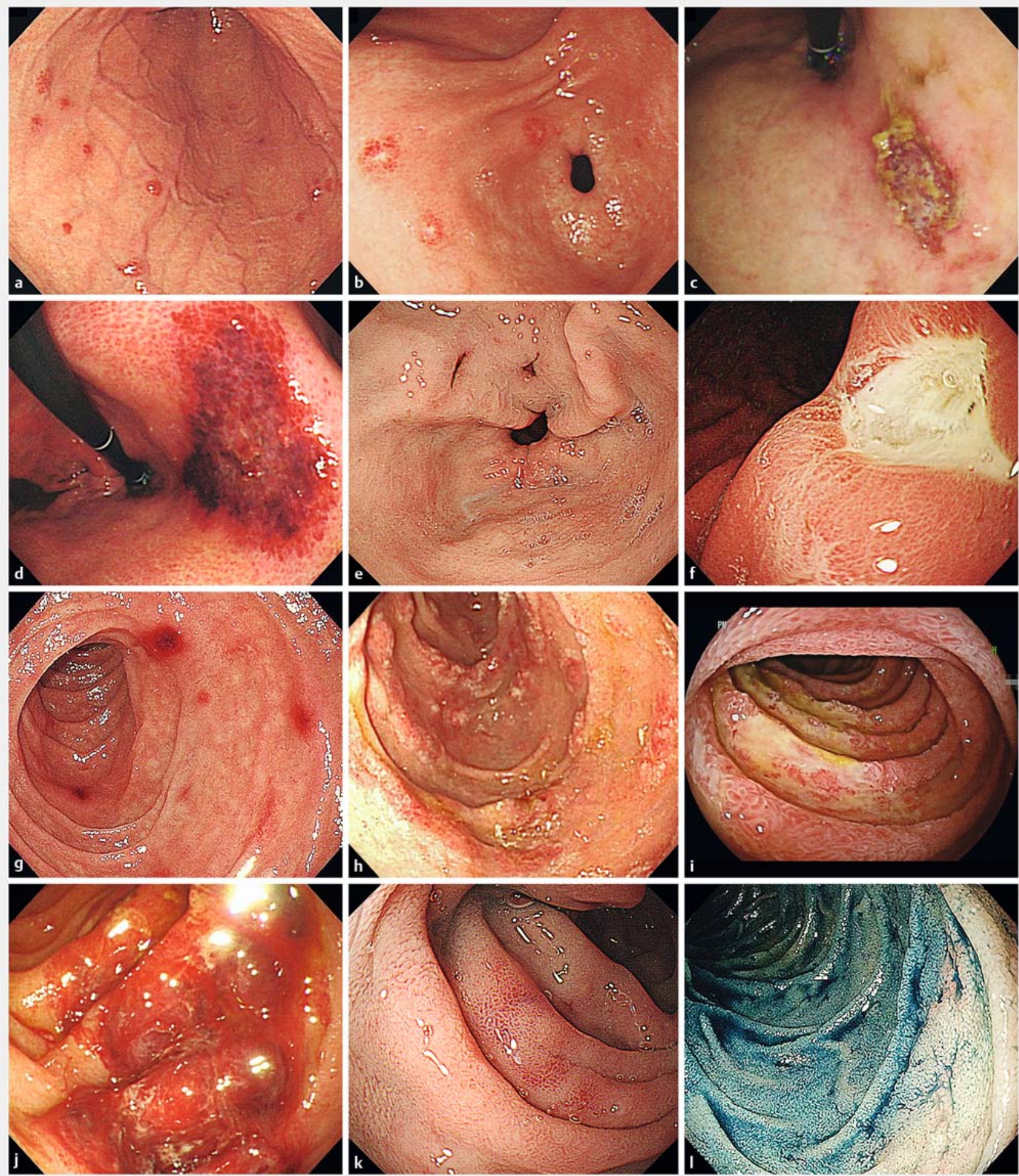

Fig. 2 Esophagogastroduodenoscopic findings in patients with $\lg A$ vasculitis $(\mathbf{a}, \mathbf{b}, \mathbf{c}, \mathbf{d}, \mathbf{g}, \mathbf{h}, \mathbf{i}, \mathbf{j})$ and those with eosinophilic granulomatosis with polyangiitis $(\mathbf{e}, \mathbf{f}, \mathbf{k}, \mathbf{I})$. a Mucosal erythemas in the middle third of the stomach. $\mathbf{b}$ Erosions in the lower third of the stomach. $\mathbf{c}$ Ulcer in the middle third of the stomach. $\mathbf{d}$ Hematoma-like protrusion in the middle third of the stomach. e Erosions in the lower third of the stomach. $\mathbf{f}$ Ulcer in the middle third of the stomach. $\mathbf{g}, \mathbf{k}$ Mucosal erythemas in the duodenum. $\mathbf{h}, \mathbf{I}$ Erosions in the duodenum. $\mathbf{i}$ Ulcers in the duodenum. j Hematoma-like protrusion in the duodenum. 

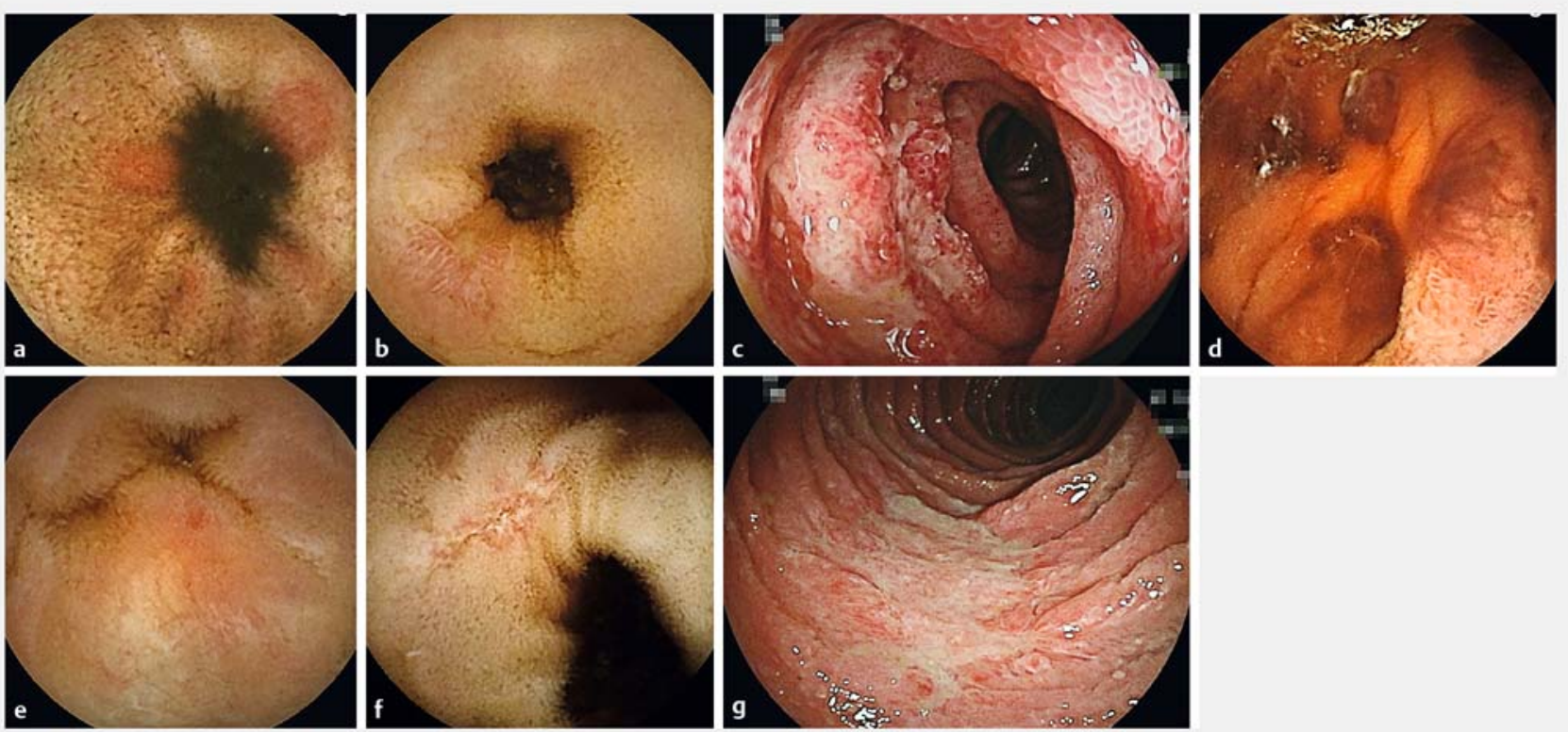

- Fig. $\mathbf{3}$ a, b, c, d Capsule or balloon-assisted endoscopic in patients with IgA vasculitis and $\mathbf{e , f}, \mathbf{g}$ those with eosinophilic granulomatosis with polyangiitis. a Mucosal erythemas in the ileum. $\mathbf{b}$ Erosions in the ileum. $\mathbf{c}$ Ulcers in the jejunum. $\mathbf{d}$ Hematoma-like protrusion in the jejunum. e Mucosal erythema in the jejunum. $\mathbf{f}$ Erosions in the ileum. $\mathbf{g}$ Ulcer in the jejunum.
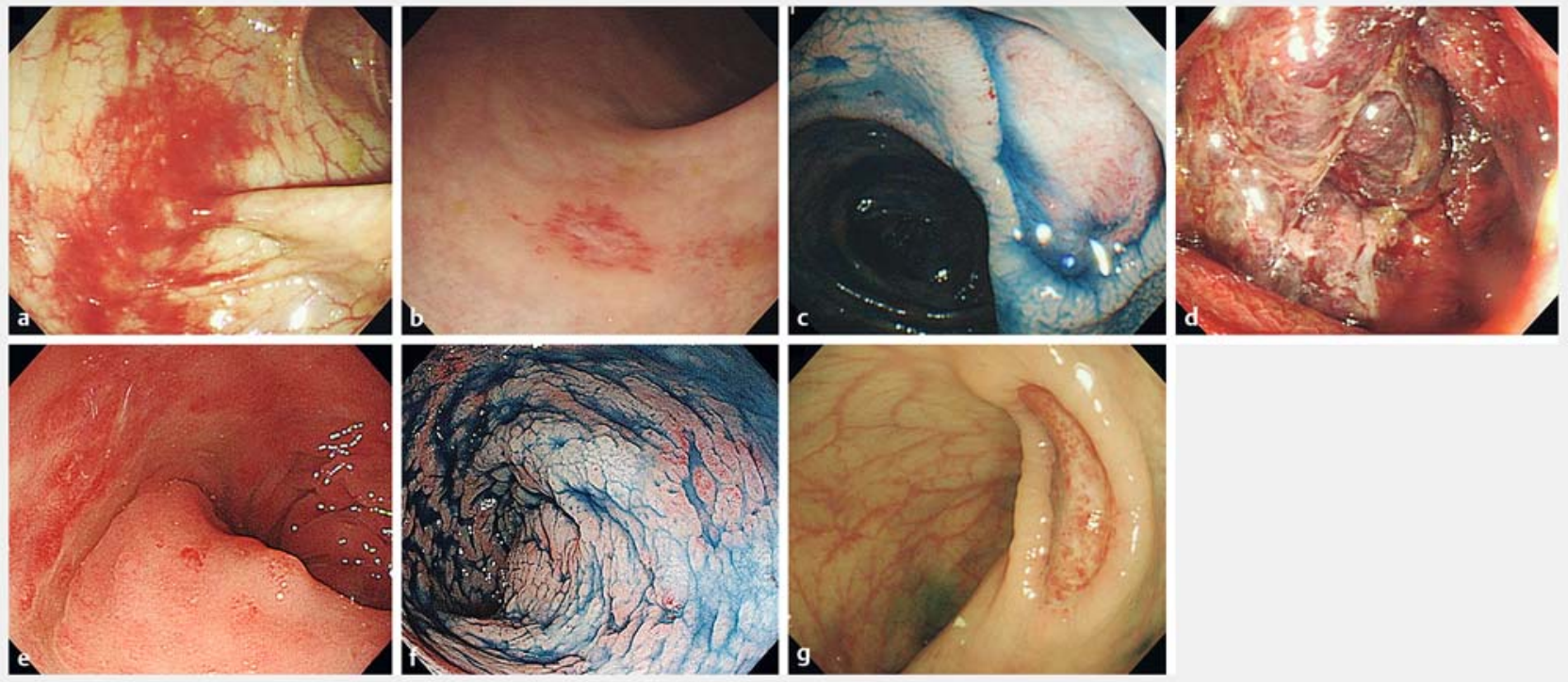

- Fig. 4 a, b, c, d Colonoscopic findings in patients with IgA vasculitis and $\mathbf{e , f}, \mathbf{g}$ those with eosinophilic granulomatosis with polyangiitis. a Mucosal erythemas in the descending colon. $\mathbf{b}$ Erosion in the rectum. $\mathbf{c}$ Ulcer in the ascending colon. $\mathbf{d}$ Hematoma-like protrusion in ascending colon. e Mucosal erythemas in the rectum. $\mathbf{f}$ Erosions in the rectum. $\mathbf{g}$ Ulcer in the rectum.

18; EGPA 17) patients, respectively. The rate of detection of the above characteristic histologic findings in all regions did not differ between the two groups (IgAV, skin 83.9\%, esophagus $0 \%$, stomach $4.4 \%$, duodenum $17.1 \%$, jejunum/ileum $14.3 \%$, colorectum $11.1 \%$; EGPA, skin $80 \%$, esophagus $12.5 \%$, stomach $23.1 \%$, duodenum $26.7 \%$, jejunum/ileum $30 \%$, colorectum $29.4 \%)$.

\section{Discussion}

In the current study, we found more frequent gastric mucosal erythema, duodenal lesions including erythema, ulcer and hematoma-like protrusion in patients with IgAV than in those with EGPA. We also revealed that incidence of jejunoileal involvement was high in both patients with IgAV and those with EGPA. 
- Table 3 Endoscopic findings in patients with gastrointestinal bleeding.

\begin{tabular}{|c|c|c|c|c|c|c|c|}
\hline Cases & Age/sex & $\begin{array}{l}\text { Clinical } \\
\text { diagnosis }\end{array}$ & $\begin{array}{l}\text { Signs of gastroin- } \\
\text { testinal bleeding }\end{array}$ & EGD findings & $\begin{array}{l}\text { CE or BAE } \\
\text { findings }\end{array}$ & $\begin{array}{l}\text { Colonoscopic } \\
\text { findings }\end{array}$ & $\begin{array}{l}\text { Endoscopic } \\
\text { hemostasis }\end{array}$ \\
\hline 1 & $83 / \mathrm{M}$ & $\lg A V$ & Hematemesis & $\begin{array}{l}\text { Mucosal erythe- } \\
\text { mas and hema- } \\
\text { toma-like pro- } \\
\text { trusions (D) }\end{array}$ & Ulcers & Normal & NP \\
\hline 2 & $64 / F$ & $\operatorname{IgAV}$ & Hematochezia & Normal & $\begin{array}{l}\text { Mucosal erythe- } \\
\text { mas and erosions }\end{array}$ & Normal & NP \\
\hline 3 & $25 / M$ & IgAV & Hematochezia & $\begin{array}{l}\text { Mucosal erythe- } \\
\text { mas (D) }\end{array}$ & $\begin{array}{l}\text { Mucosal erythe- } \\
\text { mas, erosions } \\
\text { and hematoma- } \\
\text { like protrusions }\end{array}$ & $\begin{array}{l}\text { Mucosal erythemas } \\
\text { and erosions }\end{array}$ & NP \\
\hline 4 & $20 / \mathrm{M}$ & $\operatorname{IgAV}$ & Hematochezia & $\begin{array}{l}\text { Mucosal erythe- } \\
\text { mas (D) }\end{array}$ & NE & $\begin{array}{l}\text { Mucosal erythemas, } \\
\text { erosions and hema- } \\
\text { toma-like protru- } \\
\text { sions }\end{array}$ & NP \\
\hline 5 & $78 / \mathrm{M}$ & IgAV & Melena, anemia & $\begin{array}{l}\text { Ulcers (E, S, D), } \\
\text { and hematoma- } \\
\text { like protrusions } \\
\text { (D) }\end{array}$ & NE & $\mathrm{NE}$ & NP \\
\hline 6 & $55 / M$ & $\lg A V$ & Melena & $\begin{array}{l}\text { Mucosal erythe- } \\
\text { mas and ulcers } \\
(\mathrm{S}, \mathrm{D})\end{array}$ & Ulcers & Normal & NP \\
\hline 7 & $73 / F$ & $\lg A V$ & Anemia & Normal & NE & $\mathrm{NE}$ & NP \\
\hline 8 & $53 / \mathrm{M}$ & $\lg A V$ & Hematochezia & Normal & $\begin{array}{l}\text { Mucosal erythe- } \\
\text { mas, erosions } \\
\text { and ulcers }\end{array}$ & Normal & NP \\
\hline 9 & $68 / \mathrm{F}$ & $\operatorname{IgAV}$ & Hematochezia & $\begin{array}{l}\text { Ulcers and he- } \\
\text { matoma-like } \\
\text { protrusions (D) }\end{array}$ & $\begin{array}{l}\text { Mucosal erythe- } \\
\text { mas, ulcers and } \\
\text { hematoma-like } \\
\text { protrusions }\end{array}$ & Normal & NP \\
\hline 10 & $61 / \mathrm{M}$ & EGPA & Hematochezia & Normal & Ulcers & $\begin{array}{l}\text { Mucosal erythemas } \\
\text { and ulcers }\end{array}$ & NP \\
\hline 11 & $27 / \mathrm{M}$ & EGPA & Hematochezia & Ulcers (S) & NE & Mucosal erythemas & NP \\
\hline
\end{tabular}

IgAV, previously referred to as Henoch-Schönlein purpura, is a systemic small-vessel vasculitis that develops via an allergic mechanism and is characterized by purpura associated with leukocytoclastic vasculitis [2]. Gastrointestinal symptoms occur in up to $85 \%$ of patients with IgAV [14]. Therefore, endoscopy has been used to detect the gastrointestinal involvement in IgAV. Nishiyama et al. [8] reported that gastrointestinal involvement under EGD and colonoscopy was observed in seven of 10 cases $(70 \%)$ in the stomach and in the duodenum, five cases (50\%) in the terminal ileum, and six cases (60\%) in the colon. In a report of seven cases by Esaki et al. [9], involvement with IgAV was found in two cases $(28.6 \%)$ in the stomach, six cases $(85.7 \%)$ in the duodenum, six cases $(85.7 \%)$ in the ileum, and four cases $(57.1 \%)$ in the colon.

On the other hand, EGPA, formerly known as Churg-Strauss syndrome, is a multisystem disorder characterized by allergic granulomatosis and necrotizing vasculitis developing after the appearance of peripheral and tissue eosinophilia [2,6]. The gastrointestinal tract is involved in approximately $20 \%$ to $50 \%$ of patients with EGPA. Pagnoux C et al. [15] reported that incidence of gastrointestinal involvement using EGD and colonoscopy was 17 of 62 cases ( $27 \%$ ) using EGD and six of 62 cases (10\%) using colonoscopy. In those studies, however, the entire gastrointestinal tract was not examined by endoscopy. To our knowledge, no previous studies have examined entire gastrointestinal tracts in patients with IgAV or EGPA using EGD, CE, BAE and colonoscopy.

Our results showed that incidence of esophago-gastric involvement was low, that of colorectal involvement was intermediate, and that of jejunoileal involvement was high in patients with both diseases. In addition, incidence of duodenal involvement was higher in patients with IgAV than in those with 

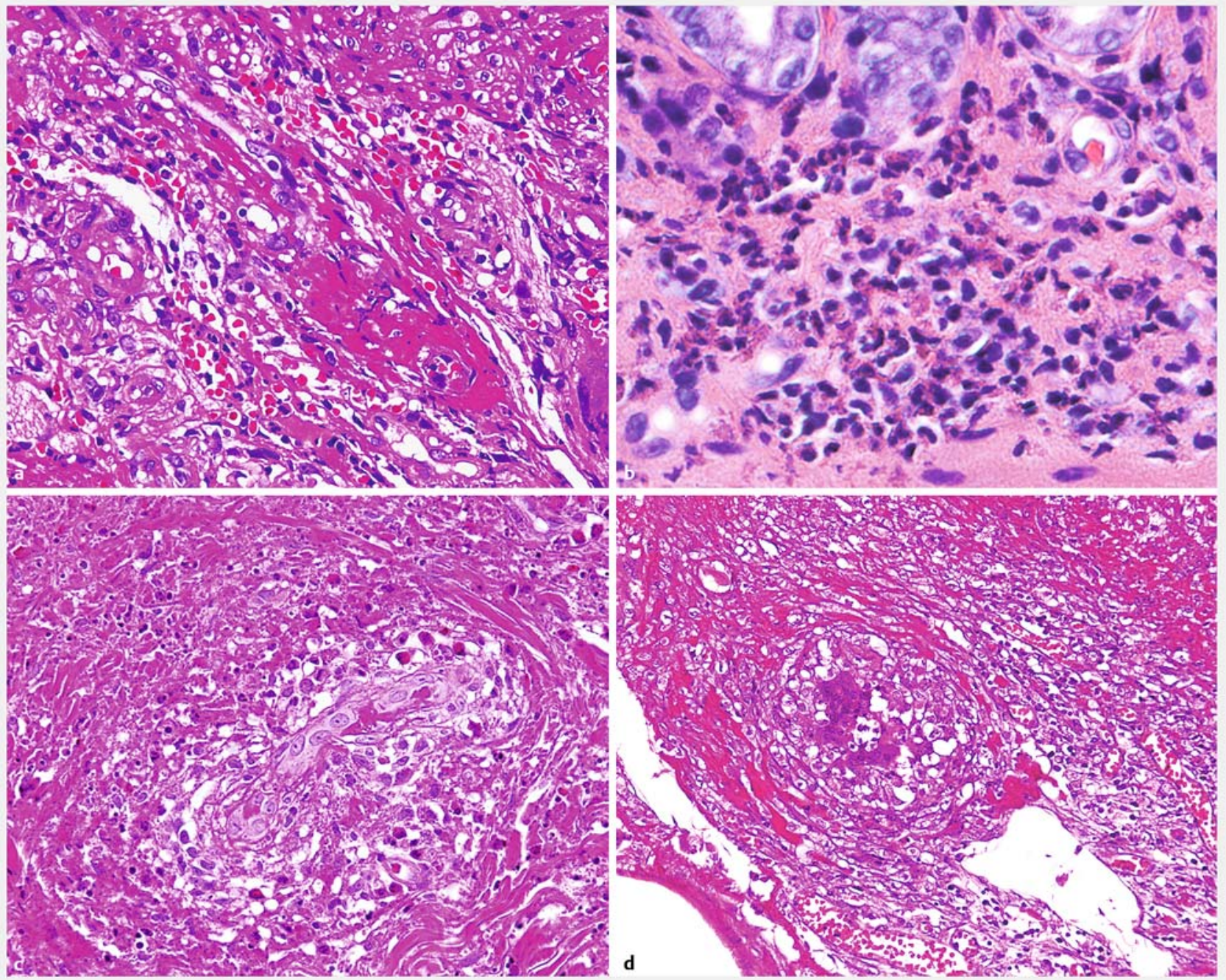

Fig. 5 Characteristic histologic findings in a IgA vasculitis and b, c, d eosinophilic granulomatosis with polyangiitis. a Leukocytoclastic vasculitis. b Eosinophilic infiltration. c Small-sized vessel vasculitis. d Extravascular granuloma.

EGPA. The previously reported incidence of esophago-gastric and colorectal involvement was unstable, because the number of cases in the previous studies is small. However, our results suggested that IgAV seems to involve predominantly the small bowel including the duodenum.

Endoscopic findings of IgAV were first reported in 1973 [16]. Akadamar et al. [16] showed that the main endoscopic findings of IgAV in the stomach and duodenum were mucosal congestion, redness, petechial, multiple ulcers, nodular change and hematoma-like protrusion. In another study, it thus seems likely that multiple irregular ulcers in the second portion are characteristic of duodenal involvement in IgAV [9]. As for colonoscopic findings in patients with IgAV, only a few case series or reports are available. Zhang et al. [7] showed that erythema, edema and petechia were common in the colon. There have been several cases regarding the diagnostic role of CE in patients with IgAV. The main endoscopic findings were erythema throughout the small bowel, and severe erosions or ulcers were found in some cases [17-21]. In the current study, we also confirmed similar findings in our patients. Thus, the above-mentioned endoscopic findings are considered characteristic in the entire gastrointestinal tract including the jejunoileum of patients with IgAV.

As for patients with EGPA, only a few studies with endoscopic findings are available, probably because most of the gastrointestinal lesions previously discussed have been in patients with gastrointestinal tract perforation [22, 23]. In such previous studies, ulcers or erythematous and nodular mucosa in the stomach, and/or ulcers or multiple small polyps in the duodenum were identified $[15,24]$. In some case reports, CE or BAE revealed that erosions, submucosal edema with lymphangiectasias and erythema, or ulcers of various shapes, such as multiple punched-out, small or irregular ulcers, were seen in the jejunum and ileum [25-28]. Colonoscopy showed mucosal erythema, erosion, ulceration, dark red sign or various red flares $[10,29]$. In the current study, we also confirmed similar find- 
ings in our patients. Thus, the above-mentioned endoscopic findings in the entire gastrointestinal tract seem characteristic in EGPA.

In the current investigation, we compared endoscopic findings between patients with IgAV and those with EGPA. As a result, mucosal erythema in the stomach, and mucosal erythema, ulcer, and hematoma-like protrusion in the duodenum were seen more frequently in patients with IgAV than in those with EGPA. Incidence of mucosal erythema, erosion, ulcer or hematoma-like protrusion in the jejunum/ileum and colorectum was no different between the two groups. In a previous study regarding EGD findings in primary vasculitis, the duodenum was the most frequently involved site [30]. Also, EGD findings including erosion, petechiae, submucosal hemorrhage or ulcers were seen in 142 of 148 patients (95.9\%). However, that study did not clarify what EGD findings are characteristic of each disease.

To date, no previous studies have compared characteristic endoscopic findings between patients with IgAV and EGPA. In the current study, we compared the endoscopic findings between the two groups. As a consequence, we found that incidence of jejunoileal involvement was high in both groups, and we also presume that gastric mucosal erythema, and mucosal erythema, ulcer, and hematoma-like protrusion in the duodenum may be characteristic endoscopic signs for IgAV.

There have only been a few studies on frequency of gastrointestinal bleeding in patients with IgAV and EGPA. In previous studies, clinical signs of gastrointestinal bleeding were found in $17 \%$ to $49 \%$ of patients with IgAV and $9 \%$ of patients with EGPA [7-9,31,32]. Our results showed that frequency of gastrointestinal bleeding was $27 \%$ (9 of 33) in patients with IgAV and $11 \%$ ( 2 of 19 patients) in patients with EGPA. We can also confirm that gastrointestinal involvement of IgAV and EGPA favorably responds to therapy regardless of presence of bleeding. There have been several case series showing fair to good response of gastrointestinal lesions in the diseases observed by endoscopy $[7,20,28,29]$. It thus seems likely that medical treatment is the first choice of treatment for gastrointestinal involvement in IgAV and EGPA.

We can also confirm that incidence of characteristic histologic findings in the gastrointestinal tract was lower compared with that of the skin. Histopathological findings of IgAV are characterized by leukocytoclastic vasculitis [4]. Nishiyama et al. [8] reported that all 11 biopsy specimens from EGD and colonoscopy in patients with IgAV showed nonspecific inflammatory cell infiltration and diagnoses were made from skin biopsies. The main histopathological findings in EGPA are extravascular granulomas, small and medium-sized vessels vasculitis, and eosinophilic infiltrates [11]. These findings are rarely seen on biopsy sections. This was probably because most biopsies are limited to the superficial portion of the mucosa and cannot reach the deeper vessels. A vasculitis diagnosis should be made with consideration of all clinical and pathologic information, but gastrointestinal involvement in patients with vasculitis may be life-threatening because of complications such as severe ischemia, infarction, and perforation. Therefore, accurate diagnosis and early treatment is important to avoid fatal out- comes, and endoscopic examination helps to define the site and extent of gastrointestinal involvement.

The current study has some limitations. We were not able to examine the entire gastrointestinal tract in all the patients diagnosed as having IgAV or EGPA from 2008 to 2017. Considering the rarity of the disease, we consider that our 52 patients are a decent number on which to base a study on IgAV and EGPA. In addition, this was retrospective study with a small sample size. Additional prospective studies of large cohorts are, therefore, needed in the near future.

\section{Conclusion}

In conclusion, our study revealed frequent duodenal involvement, gastric mucosal erythema, and duodenal mucosal erythema, ulcer, and hematoma-like protrusion in patients with IgAV. We also foound that incidence of jejunoileal involvement was high in patients with IgAV and those with EGPA. These findings support use of small-bowel endoscopy as a helpful procedure for diagnosis of small-bowel lesions in IgAV or EGPA.

\section{Competing interests}

None

References

[1] Jennette JC, Falk RJ, Andrassy K et al. Nomenclature of systemic vasculitides. Proposal of an international consensus conference. Arthritis Rheum 1994; 37: 187-192

[2] Jennette JC, Falk RJ, Bacon PA et al. 2012 revised International Chapel Hill Consensus Conference Nomenclature of Vasculitides. Arthritis Rheum 2013; 65: 1-11

[3] Louie CY, DiMaio MA, Charville GW et al. Gastrointestinal tract vasculopathy: clinicopathology and description of a possible "new entity" with protean features. Am J Surg Pathol 2018; 42: 866-876

[4] Mills JA, Michel BA, Bloch DA et al. The American College of Rheumatology 1990 criteria for the classification of Henoch-Schönlein purpura. Arthritis Rheum 1990; 33: 1114-1121

[5] Martinez-Frontanilla LA, Haase GM, Ernster JA et al. Surgical complications in Henoch-Schönlein Purpura. J Pediatr Surg 1984; 19: 434 436

[6] Chumbley LC, Harrison EG Jr, DeRemee RA. Allergic granulomatosis and angiitis (Churg-Strauss syndrome). Report and analysis of 30 cases. Mayo Clin Proc 1977; 52: 477-484

[7] Zhang Y, Huang X. Gastrointestinal involvement in Henoch-Schönlein purpura. Scand J Gastroenterol 2008; 43: 1038 - 1043

[8] Nishiyama R, Nakajima N, Ogihara A et al. Endoscope images of Schönlein-Henoch purpura. Digestion 2008; 77: 236-241

[9] Esaki M, Matsumoto T, Nakamura $S$ et al. gastrointestinal involvement in Henoch-Schönlein purpura. Gastrointest Endosc 2002; 56: $920-$ 923

[10] Tsurikisawa N, Oshikata C, Tsuburai T et al. Th17 cells reflect colon submucosal pathologic changes in active eosinophilic granulomatosis with polyangiitis. BMC Immunol 2015; 16: 75

[11] Gioffredi A, Maritati F, Oliva E et al. Eosinophilic granulomatosis with polyangiitis: an overview. Front Immunol 2014; 5: 549 
[12] Fries JF, Hunder GG, Bloch DA et al. The American College of Rheumatology 1990 criteria for the classification of vasculitis. Summary. Arthritis Rheum 1990; 33: 1135-1136

[13] Masi AT, Hunder GG, Lie JT et al. The American College of Rheumatology 1990 criteria for the classification of Churg-Strauss syndrome (allergic granulomatosis and angiitis). Arthritis Rheum 1990; 33: $1094-1100$

[14] Szer IS. Henoch-Schönlein purpura: when and how to treat. J Rheumatol 1996; 23: $1661-1665$

[15] Pagnoux C, Mahr A, Cohen P et al. Presentation and outcome of gastrointestinal involvement in systemic necrotizing vasculitides: analysis of 62 patients with polyarteritis nodosa, microscopic polyangiitis, Wegener granulomatosis, Churg-Strauss syndrome, or rheumatoid arthritis-associated vasculitis. Medicine (Baltimore) 2005; 84: 115 128

[16] Akdamar K, Agrawal NM, Varela PY. The endoscopic appearance of anaphylactoid purpura. Gastrointest Endosc 1973; 20: 68-69

[17] Kishikawa H, Nishida J, Takarabe S et al. "Circular reddish lesions": a possibly characteristic endoscopic finding in Henoch-Schönlein purpura. Endoscopy 2013; 45: E33-E34

[18] Skogestad E. Capsule endoscopy in Henoch-Schonlein purpura. Endoscopy 2005; 37: 189

[19] Stancanelli B, Vita A, Vinci M et al. Bleeding of small bowel in HenochSchönlein syndrome: the successful diagnostic role of video capsule endoscopy. Am J Med 2006; 119: 82 - 84

[20] Preud'Homme DL, Michail S, Hodges C et al. Use of wireless capsule endoscopy in the management of severe Henoch-Schonlein purpura. Pediatrics 2006; 118: $904-906$

[21] Ichikawa R, Hosoe N, Imaeda $\mathrm{H}$ et al. Evaluation of small-intestinal abnormalities in adult patients with Henoch-Schönlein purpura using video capsule. Endoscopy 2011; 43: 162-163

[22] Assmann G, Molinger M, Pfreundschuh M et al. Gastrointestinal perforation due to vasculitis at primary diagnosis of eosinophilic granu- lomatosis with polyangiitis (EGPA) despite a high dose glucocorticosteroids treatment. Springerplus 2014; 3: 404

[23] Ohnuki Y, Moriya Y, Yutani S et al. Eosinophilic granulomatosis with polyangiitis (Churg-Strauss Syndrome) complicated by perforation of the small intestine and cholecystitis. Intern Med 2018; 57: 737-740

[24] Franco DL, Ruff K, Mertz L et al. Eosinophilic granulomatosis with polyangiitis and diffuse gastrointestinal involvement. Case Rep Gastroenterol 2014; 8: 329-336

[25] Sánchez R, Aparicio JR, Baeza T et al. Capsule endoscopy diagnosis of intestinal involvement in a patient with Churg-Strauss syndrome. Gastrointest Endosc 2006; 6: 1082 - 1084

[26] Beye B, Lesur G, Claude P et al. Small bowel involvement documented by capsule endoscopy in Churg-Strauss syndrome. Pan Afr Med J 2015; 22: 41

[27] Suzuki T, Matsushima M, Arase Y et al. Double-balloon endoscopy-diagnosed multiple small intestinal ulcers in a Churg-Strauss syndrome patient. World J Gastrointest Endosc 2012; 4: 194-196

[28] Kawasaki K, Eizuka M, Murata O et al. Eosinophilic granulomatosis with polyangiitis involving the small intestine: radiographic and endoscopic findings. Endoscopy 2015; 47: $492-494$

[29] Yoshioka T, Kusaka T, Seno H. Colonic findings in Churg-Strauss syndrome. Endoscopy 2013; 45: 185-186

[30] Gong EJ, Kim do H, Chun JH et al. Endoscopic findings of upper gastrointestinal involvement in primary vasculitis. Gut Liver 2016; 10 : $542-548$

[31] Audemard-Verger A, Terrier B, Dechartres A et al. Characteristics and Management of IgA vasculitis (Henoch-Schönlein) in adults: data from 260 patients included in a French multicenter retrospective survey. Arthritis Rheumatol 2017; 69: $1862-1870$

[32] Durel CA, Berthiller ], Caboni S et al. Long-term followup of a multicenter cohort of 101 patients with eosinophilic granulomatosis with polyangiitis (Churg-Strauss). Arthritis Care Res (Hoboken) 2016; 68: $374-387$ 